\title{
ETUDE DE LA REPRODUCTION DE L'ABLE DE HECKEL (LEUCASPIUS DELINEATUS) DANS UN ETANG BRETON
}

\author{
Anne-Isabelle CASSOU et H. LE LOUARN
}

Laboratoire d'Ecologie Hydrobiologique-INRA, 65 rue de St-Brieuc - 35042 RENNES Cedex

\section{RÉSUMÉ}

Une étude du recrutement de l'able de Heckel (Leucaspius delineatus), pour l'année 1990, a été menée dans un bassin vidangeable de $500 \mathrm{~m}^{2}$ en BRETAGNE au RHEU (ouest de RENNES).

Plus de 200.000 œufs ont été collectés sur supports artificiels (plaques en PVC), et leur évolution suivie au cours du temps, ainsi que la maturation des ovaires.

La reproduction a débuté mi-mai pour une température moyenne de $20^{\circ} \mathrm{C}$, et cessé mijuillet quand les gonades s'atrophiaient. La fraie, de type "fractionnée asynchrone", a lieu lorsqu'une proportion suffisante (20\%) d'ovocytes en fin de vitellogénèse est atteinte dans la gonade. Trois maxima de pontes ont été observés (fin mai, mi-juin, fin juin), mais seule la première série d'œufs présentait un nombre important d'éclosions (plus de $50 \%$ en aquarium), alors que le taux de fécondation était identique pour toute la saison (60 à $80 \%$ ). A ces 3 maxima de pontes ont correspondu 3 cohortes d'alevins observées en fin de saison de reproduction.

Mots-clés : Etang, Frayère artificielle, Recrutement, Ponte fractionnée asynchrone, Leucaspius delineatus, able de Heckel.

\section{STUDY OF THE REPRODUCTION OF LEUCASPIUS DELINEATUS IN A POND (BRITTANY)}

\section{SUMMARY}

During year 1990, a study of the recruitment of Leucaspius delineatus was carried out in a drainable reservoir of $500 \mathrm{~m}^{2}$ at the RHEU (Brittany).

More than 200.000 eggs were collected on artificial (PVC) supports. Eggs evolution and ovaries maturation were studied.

Reproduction began in the middle of May for an average water temperature of $20^{\circ} \mathrm{C}$, and stopped in the middle of July when gonads were atrophied. The spawning, "portionedasynchronous-reproduction", occurred when an adequate supply $(20 \%)$ of oocytes at the end of vitellogenesis was reached in the gonad. Three maxima of egg-laying were observed (end of May, mid-June, end of June). Only the first series of eggs showed a large number of hatching (more than $50 \%$ ), although the fecundation rate was identical along the season (60 to $80 \%$ ).

These 3 maxima of egg-laying corresponded with 3 cohorts of fry observed late in the season of reproduction.

Key-words : Pond, Artificial support, Recruitment, Portioned-asynchronous-reproduction, Leucaspius delineatus. 


\section{INTRODUCTION}

L'able de Heckel (Leucaspius delineatus) est un Cyprinidé faisant souvent partie de la faune piscicole des étangs d'Europe. II se développe et se reproduit dans des conditions écologiques variables (il vit à des latitudes diverses en URSS : BREZEANU, 1968). La biologie de cette espèce a commencé à être étudiée dans les pays de l'Est, les premières données datant de 1949 (BERG, in BREZEANU, 1968).

L'able a une alimentation à base d'organismes zooplanctoniques et de larves d'insectes (PAVLOV et SMIRNOV, 1965, in BREZEANU, 1968), et atteint rapidement une taille de 5 à $10 \mathrm{~cm}$ à l'âge adulte. Sa reproduction a lieu de juin à août, à une température de 15 à $20^{\circ} \mathrm{C}$. La femelle dépose un ou plusieurs rubans d'œufs sur les bords inférieurs des grandes feuilles, autour des tiges ou des objets flottants. Le frai est protégé par une structure primitive, en forme de nid, composée de végétaux et gardée par le mâle qui assure sa ventilation jusqu'à l'éclosion (PHILLIPPART et VRAUKEN, 1983). La ponte est soupçonnée de type "fractionnée asynchrone" (BREZEANU, 1968), une même femelle produisant alors plusieurs séries d'œufs au cours de la saison de reproduction. Ce phénomène est encore mal connu chez cette espèce, alors que plusieurs Téiéostéens utilisant la même stratégie de reproduction ont déjà été étudiés (WALLACE et SELMAN, 1981).

Les pontes de l'able peuvent être observées relativement facilement grâce à sa faible sélectivité pour le substrat (il accepte les frayères artificielles), sur lequel les œufs sont collés en chapelets. Le dénombrement aisé des pontes dans l'étang a été associé à l'analyse histologique de la vitellogénèse.

Cette étude a été menée afin d'estimer l'intérêt de l'utilisation de l'able comme poissonfourrage pour le brochet. En effet, sa petite taille, même à l'âge adulte, et sa grande prolificité, en font une espèce particulièrement intéressante.

\section{MATÉRIEL ET MÉTHODE}

\section{Le Milieu}

L'étude s'est déroulée dans un étang artificiel vidangeable de $500 \mathrm{~m}^{2}(50 \times 10 \mathrm{~m})$, sur le domaine du RHEU appartenant à I'INRA, en ILLE-ET-VILAINE (35). Ce bassin est alimenté par une rivière, la FLUME, et se caractérise par une eau peu profonde (maximum $1,20 \mathrm{~m}$ ) de transparence réduite, et un fond vaseux. La température de l'eau a été relevée en continu à $20 \mathrm{~cm}$ de profondeur et le $\mathrm{pH}$ noté chaque semaine. Au cours de l'année, les températures ont varié de 2 à $29^{\circ} \mathrm{C}$, l'alcalinité de 0,9 à 1,2 méq./l et le pH de 8 à 11 . Ces fortes valeurs de $\mathrm{pH}$ trouvent leur explication dans la faible pluviométrie du printemps qui a entraîné, lors du remplissage du bassin, un apport important de nitrates et de phosphates. L'eau s'est également enrichie lors des pompages effectués pour le maintien de son niveau, avec pour conséquence directe un développement important d'algues filamenteuses. Les températures élevées de 23 à $26^{\circ} \mathrm{C}$ en moyenne (à $20 \mathrm{~cm}$ de profondeur) durant l'été ont favorisé principalement l'espèce Hydrodictyon reticulatum. Sur ce terrain acide, où les déséquilibres carbonatés ne peuvent être tamponnés par les apports de substrat, le pH dépend étroitement de l'intensité de l'activité photosynthétique. II s'en est suivi des valeurs très élevées du potentiel hydrogène.

\section{Les poissons}

La population de départ était constituée de 1.200 adultes élevés en bassin, à partir d'œufs collectés dans la FLUME en 1988. Ils ont été libérés dans l'étang à l'automne 1989.

L'étude de ces individus s'est effectuée du mois d'avril au mois d'août 1990. Au cours de cette période, le déroulement de la gamétogenèse a été suivi par l'observation de coupes d'ovaires et des variations du rapport gonado-somatique. Les données histologiques ont été comparées à la réalité : par collectes des pontes dans le milieu naturel sur frayères artificielles, et constatation du devenir de ce recrutement en fin d'été. 
Les 10 à 20 adultes sacrifiés lors de chaque prélèvement hebdomadaire ont été pêchés au carrelet. Ils ont été mesurés (longueur à la fourche de la nageoire caudale) et pesés. Le sexe de l'animal était reconnu à l'aspect des gonades lors de l'autopsie.

\section{Histologie}

Après fixation dans du Bouin de Hollande, et inclusion dans des blocs de paraffine, les ovaires ( 3 à 7 traités par semaine) ont été coupés en sections de $10 \mu \mathrm{m}$ et colorés à l'Azan de Heidenhaim (GABE, 1968). Pour chaque gonade, la proportion des différents types d'ovocytes a été estimée sous un projecteur "micro-macro" : une échelle micrométrique (de $32 \mathrm{~cm}$ ) traversait l'écran et les ovocytes la rencontrant étaient classés par stades, mesurés et comptés. L'examen histologique des ovaires permet de mettre en évidence les étapes successives de la vitellogénèse (KARTAS et QUIGNARD, 1984). Elles ont été définies pour l'able, dans cette étude, par rapprochement avec les travaux de BRETON et al. (1980) sur la tanche et de ZOHAR (1982) sur la truite.

Le rapport gonado-somatique, mis en parallèle avec les données histologiques, a été calculé selon la formule : Poids des gonades $\times 100 /($ Poids du poisson - Poids des gonades).

\section{Observation de la traie}

Afin d'acquérir des renseignements sur la biologie de la fraie, des supports artificiels ont été testés. GILLET (1989) ayant déjà constaté que certains Cyprinidés acceptaient de pondre sur divers matériaux, quinze plaques en PVC de format $21 \times 29,7 \mathrm{~cm}$ ontété suspendues dans l'étang le long d'un câble à $20 \mathrm{~cm}$ de profondeur.

Le nombre d'œufs a été estimé tous les deux jours sur chaque plaque.

Afin de connaître la survie des œufs sans perturbation, certaines plaques ont été laissées en place dans l'étang (fig. 1a).

Pour observer leur évolution tout en les isolant des géniteurs, d'autres frayères ont été placées dans des enceintes à armature en fer et tendues de tissus en nylon (fig. 1b).

L'utilisation d'aquariums poury déposer certaines plaques a permis d'observer les éclosions. L'eau provenant de l'étang était maintenue à une température proche de celle du milieu naturel : $18^{\circ} \mathrm{C}$ (fig. 1C).
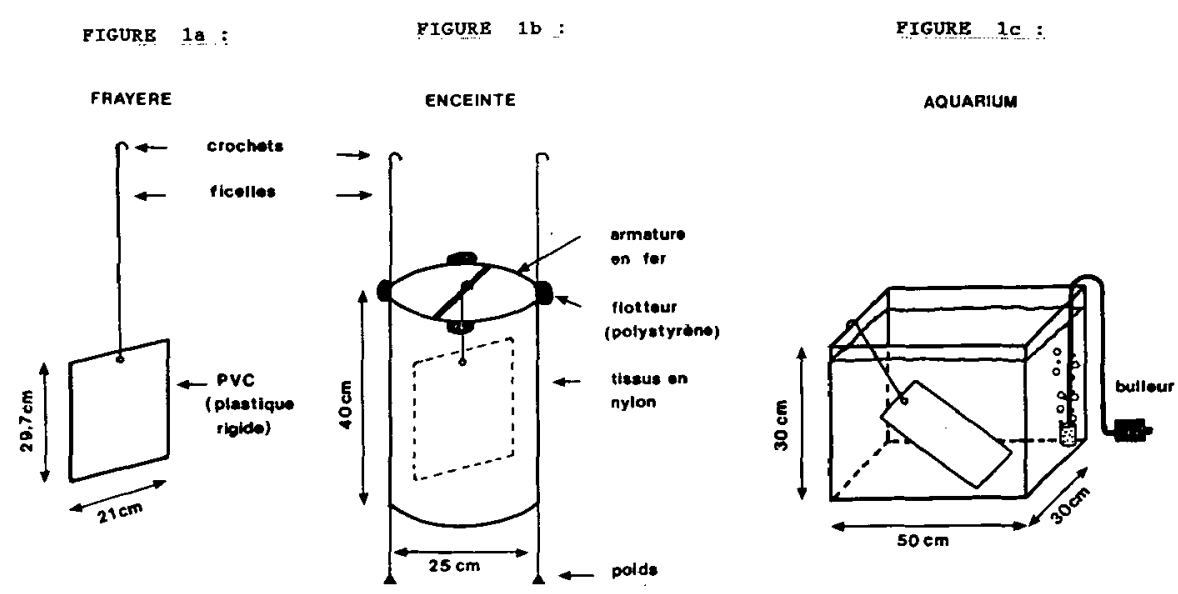

Figure 1a : Plaque en PVC suspendue à $20 \mathrm{~cm}$ de profondeur dans l'étang, et utilisée comme frayère pour la collecte des œufs d'able de Heckel.

16 : Enceinte flottant dans l'étang, permettant d'isoler une frayère des géniteurs.

1c :Disposition d'une frayère dans un aquarium pour l'observation des éclosions.

Figure 1a : PVC plate hanged at $20 \mathrm{~cm}$ of depth in the pond, and utilized as spawningground for collecting Leucaspius eggs.

1b : Floating walls in the pond, isolating spawning-ground from spawners.

1c : Spawning-ground is placed in an aquarium for the observation of hatching. 


\section{Structure de la population}

Afin d'estimer, de façon exhaustive, la densité finale de poissons en fin d'étude, le bassin a dû être vidangé au mois d'août dans une pêcherie en béton.

Les ables récupérés ont été pesés individuellement, pour séparer les jeunes des adultes. Un lot de 100 alevins a été mesuré afin d'affiner les classes de poids, et dissocier les différentes cohortes. Quelques écailles ont été prélevées pour vérifier l'âge de certains poissons selon la méthode scalimétrique (BAGLINIERE et LE LOUARN, 1987).

\section{RÉSULTATS}

\section{Dynamique de l'ovogénèse}

Une classification des stades de croissance des ovocytes d'ables a été effectuée par observations des coupes d'ovaires. Cela a permis de séparer nettement 5 périodes (fig. $2 \mathrm{f}$ ) :

- La prévitellogénèse (fig. 2a) :

Les basophiles (ovocytes sans vitellus) mesurent 0,02 à $0,4 \mathrm{~mm}$ de diamètre. Ils sont de couleur jaune sur les coupes, avec un grand noyau contenant de nombreux nucléoles à sa périphérie. Les couches folliculaires ne sont pas encore organisées.

- La vitellogénèse endogène (fig. $2 a$ et $2 b$ ) :

Les ovocytes I ont un diamètre de 0,12 à $0,56 \mathrm{~mm}$ de diamètre. Des vésicules vitellines apparaissent à la périphérie de l'ovoplasme et gagnent son centre. Ce sont les composants vitellins spécifiques synthétisés qui donnent une couleur bleutée. La zona radiata et la granulosa se structurent (en rouge).

- La vitellogénèse exogène (fig. $2 \mathrm{c}$ ) :

Les ovocytes II ont un diamètre de 0,36 à $0,86 \mathrm{~mm}$. Ils sont caractérisés par l'incorporation de vitellogénine (teinte rouge de plus en plus foncée). L'enveloppe extérieure s'épaissit.

- L'atrésie folliculaire (fig. 2d) :

La thèque se brise parfois en plusieurs endroits, l'ovocyte alors se contracte et se résorbe. Cette dégénérescence peut apparaître à divers stades de croissance et de différenciation des ovocytes.

- L'ovulation (fig. 2e) :

La ponte est imminente quand le noyau migre à la périphérie d'un ovocyte II. Après son expulsion dans la cavité ovarienne, ne reste en place que l'enveloppe folliculaire. 


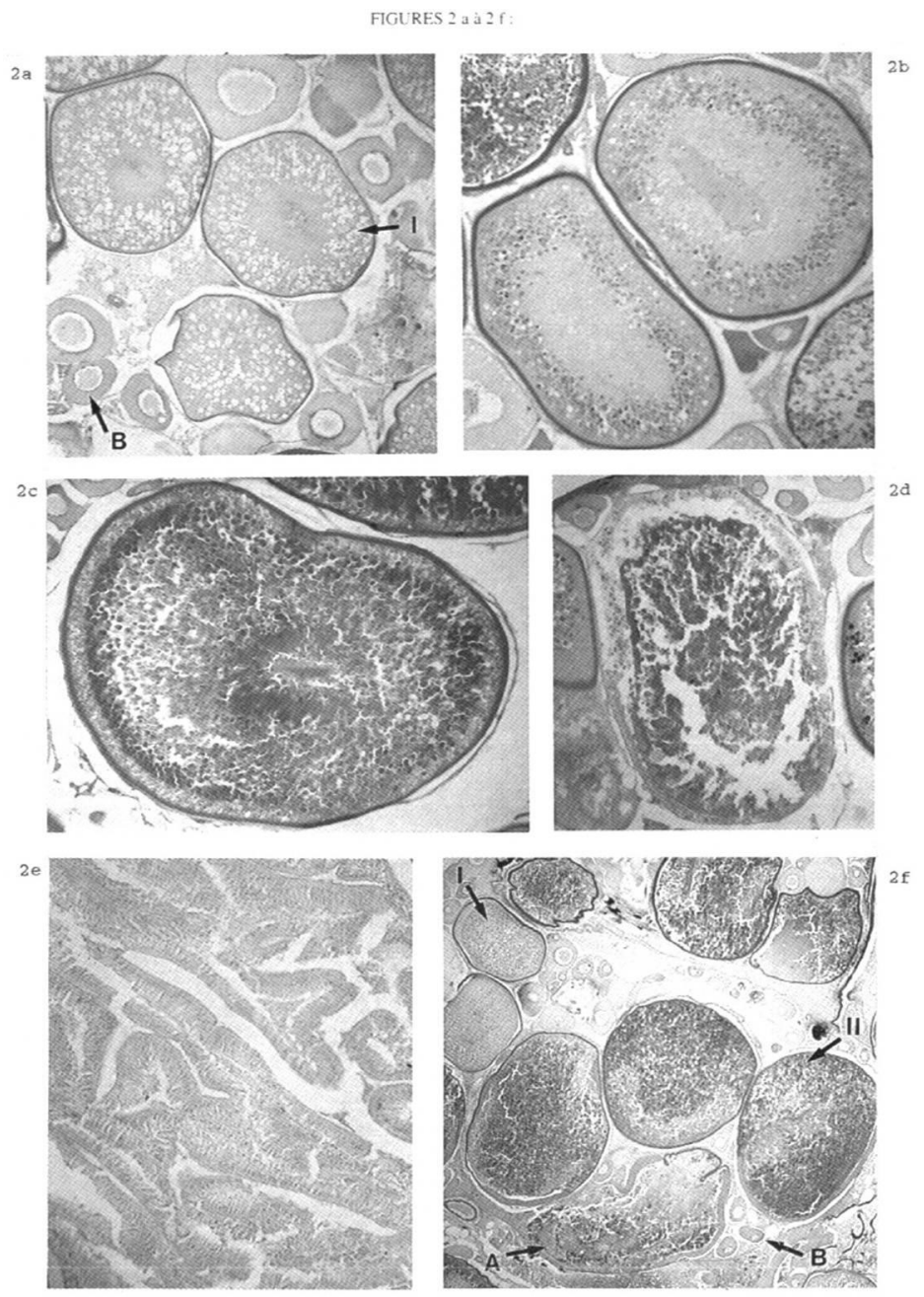

Figures 2a à $2 f$ : Coupes d'ovaires d'ables colorées à l'Azan de Heidenheim, avec des ovocytes aux différents stades de croissance.

2a : Ovocytes en prévitellogénèse ou basophiles (jaunes : B) et Ovocytes primaires (bleus : I).

2b : Ovocytes primaires avec vitellus endogène (bleu).

2c : Ovocyte secondaire avec vitellus exogène (rouge).

2d : Atrésie d'un ovocyte, pouvant apparaître à différents stades.

2e : Follicules atrésiques post-ovulatoires.

2f : Vue générale d'une coupe d'ovaire: Basophiles (B), Ovocyte primaire (I), Ovocyte secondaire (II) et Ovocyte atrésique (A).

Figures $2 a$ à $2 f$ :Sections of Leucaspius ovaries coloured with the Azan of Heidenheim, with oocytes at different stages of growth.

2a : Previtellogenic oocytes or basophiles (yellow : B) and primary oocytes (blue : I).

2b : Primary oocytes with endogenous yolk substances (blue).

2c : Secundary oocyte with exogenous yolk substances (red).

2d : Atretic oocyte, occurring at different stages.

2e $\quad$ : Postovulatory follicular cells.

2f : General view showing an ovary section : Basophiles (B), Primary oocyte (I), Secundary oocyte (II) and Atretic oocyte (A). 
Chaque vague de pontes correspond à une diminution du nombre de basophiles, qui se transforment en ovocytes I puis II. Quand une gonade contient environ $20 \%$ d'ovocytes II en fin de vitellogénèse, ils sont libérés. Mi-mai, une importante atrésie de $10 \%$ a été constatée (fig. 3a).

Le rapport gonado-somatique est resté toutefois constant jusqu'en juillet (fig. 3b). Au mois d'août, les gonades se sont atrophiées.

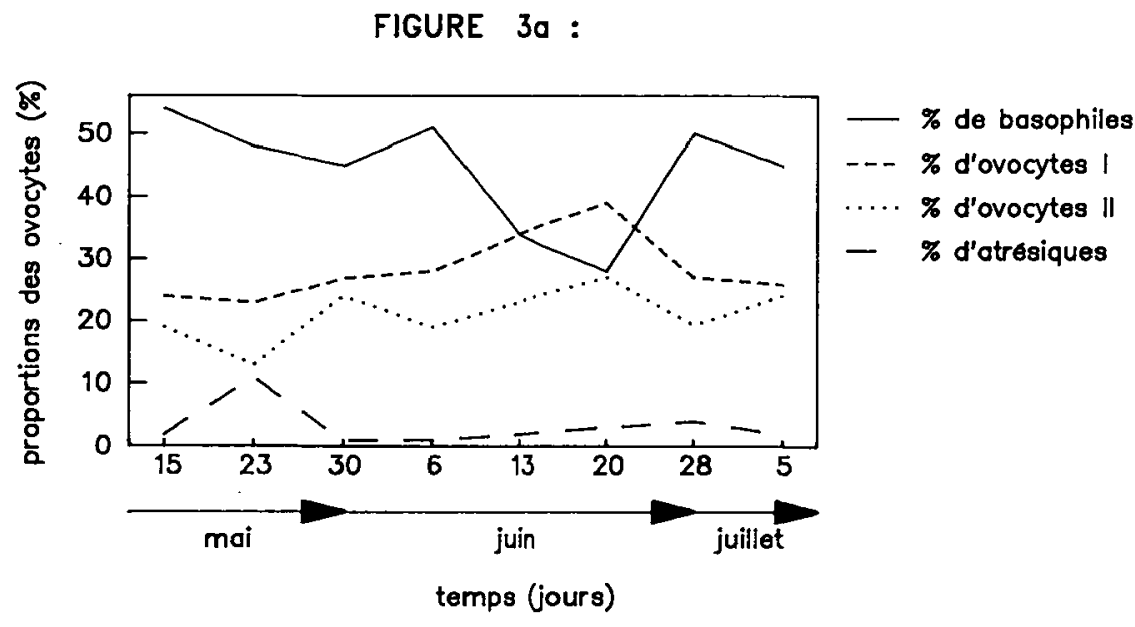

FIGURE 3b :

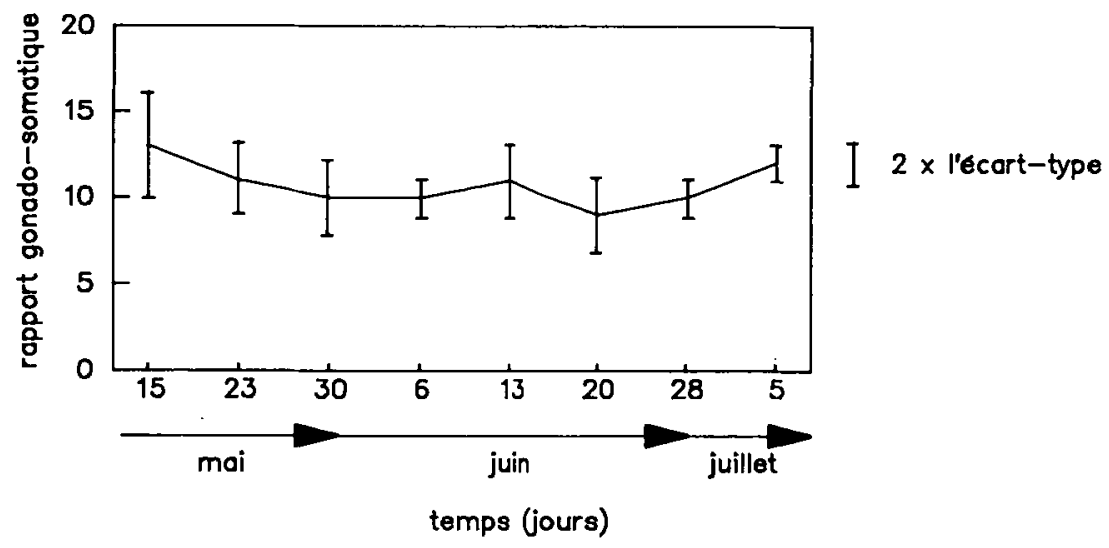

Figure 3a : Evolution de la proportion des différents stades d'ovocytes dans les gonadés d'ables femelles au cours de la période d'étude (analyse de 5 gonades chaque semaine en moyenne).

3b: Evolution du rapport gonado-somatique des ables femelles au cours de la période d'étude (analyse de 8 gonades par semaine en moyenne).

Figure 3a: Evolution of proportion of the different stages of oocytes in the female Leucaspius gonads during the studied period ( 5 gonads analysed every week in average).

3b: Evolution of gonado-somatic rapport of female Leucaspius during the studied period ( 8 gonads analysed every week in average). 


\section{Fécondité}

En milieu naturel, les pontes ont débuté le 17 mai pour une température moyenne de $20^{\circ} \mathrm{C}$ et un pH inférieur à 10 (fig. $4 a$ et $4 \mathrm{~b}$ ), et se sont arrêtées après le 16 juillet.

FIGURE 4a :

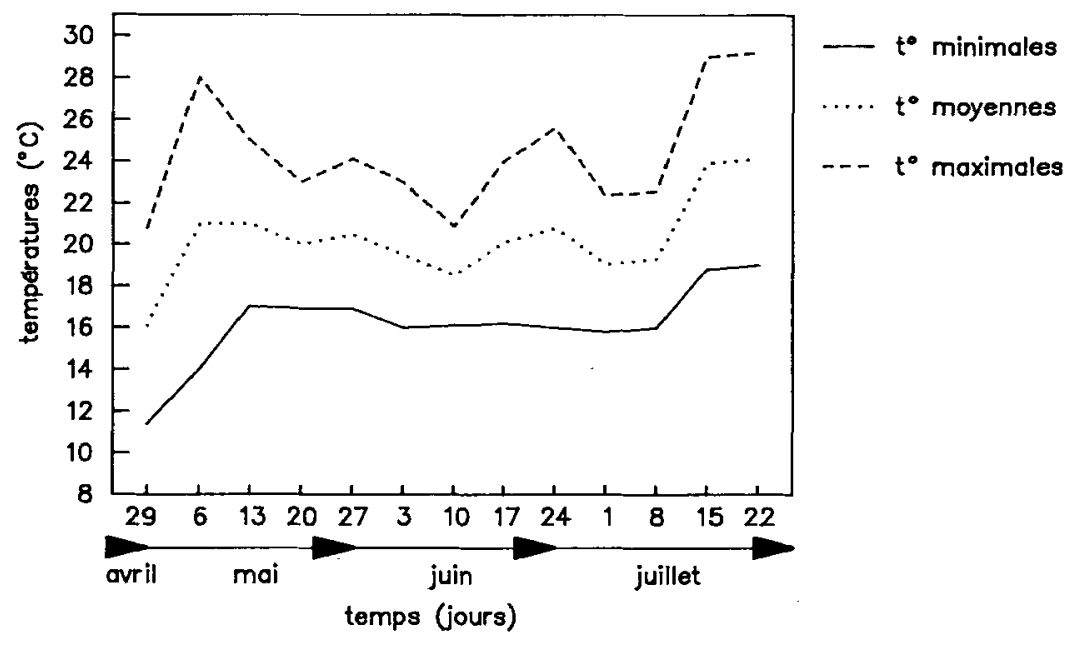

FIGURE 4b :

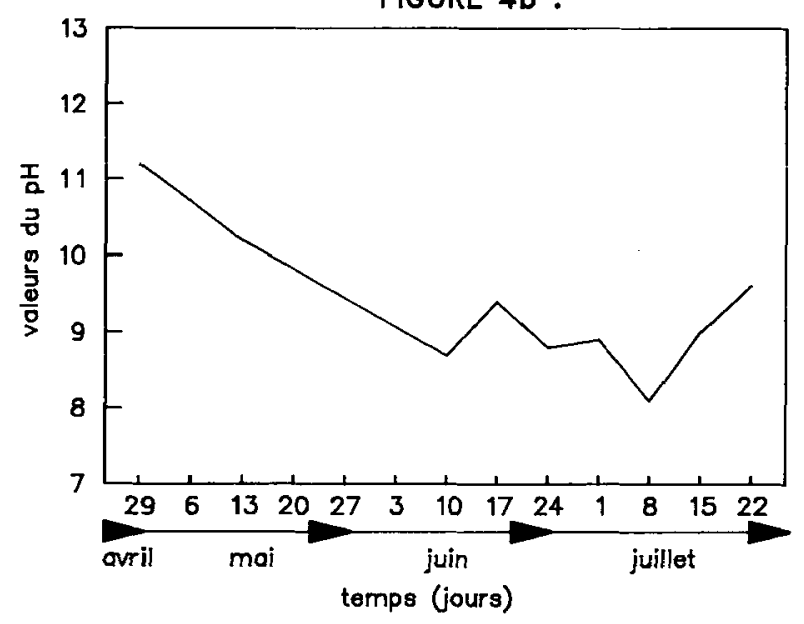

Figure 4a : Evolution au cours de la période d'étude des températures moyenne, minimale et maximale de l'eau (relevées dans l'étang à $20 \mathrm{~cm}$ de profondeur).

4b : Evolution du pH de l'eau au cours de la période d'étude.

Figure 4a : Evolution during the studied period of the average, minimal and maximal water temperatures (recorded in the pond at $20 \mathrm{~cm}$ of depth).

$4 \mathrm{~b}$ : Evolution of water $\mathrm{pH}$ during the studied period.

La période de reproduction a donc duré 2 mois pour l'année 1990 et a montré 3 maxima de pontes (fig. 5 ) : le 28 mai, le 15 juin et le 28 juin. Les chapelets contenaient une centaine d'œufs (soit 12 œufs par centimètre environ) mesurant $1,4 \mathrm{~mm}( \pm 0,1)$ en moyenne. 
Pour chaque série de pontes, le nombre d'ovules $( \pm \sigma)$ augmentait avec le poids des ovaires, et sans doute avec le nombre de femelles frayant : 1761 ( \pm 805 ) œufs par plaque, puis $2515( \pm 998)$ et $3468( \pm 1084)$ respectivement.

En milieu confiné, malgré une fécondabilité potentielle de 60 à $80 \%$ et une durée stable d'embryogénèse de $110( \pm 19)$ degrés-jour, seule la première série de pontes a présenté un taux de naissance de plus de $50 \%$ (fig. 6).

Mais, que ce soit en aquarium ou en enceinte, des proliférations d'algues et de champignons sur les pontes ont été observées, détruisant des chapelets entiers d'œufs et introduisant ainsi un biais dans les résultats.

FIGURE 5 :

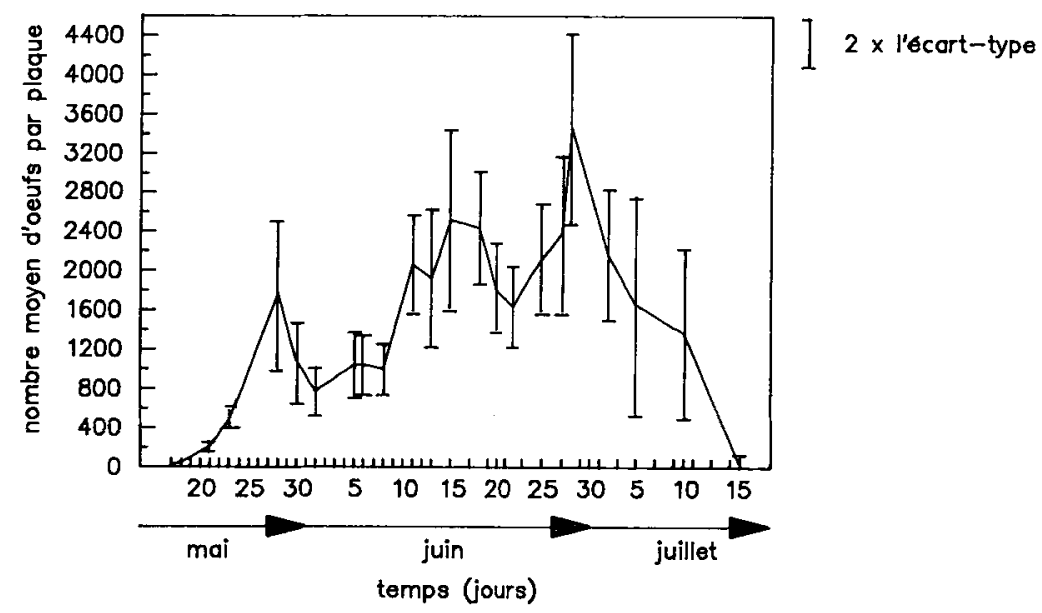

FIGURE 6 :

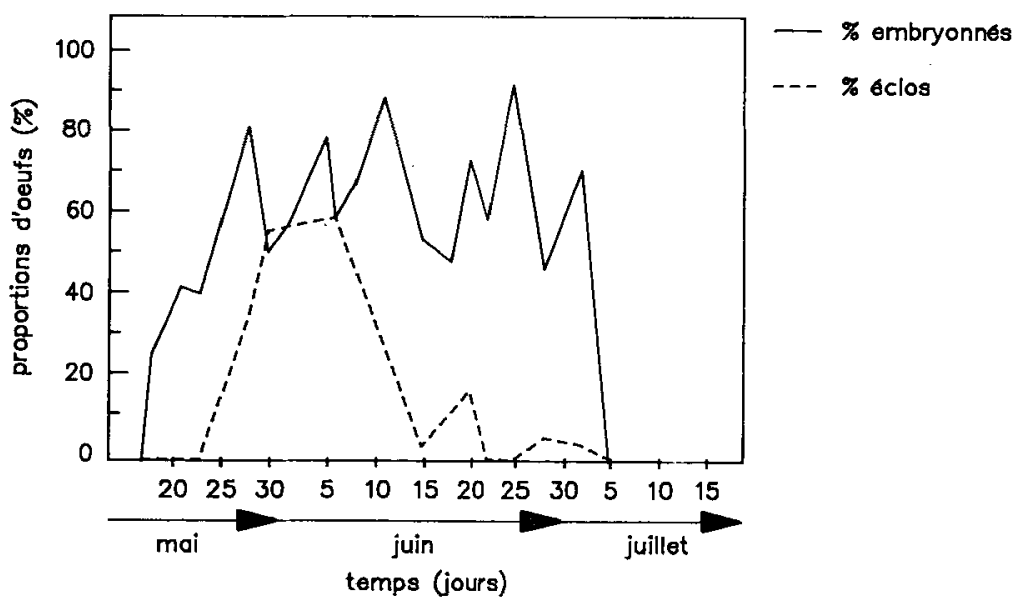

Figure 5 : Evolution du nombre moyen d'œufs d'ables pondus par plaque au cours de la période d'étude.

Figure 5 : Evolution of the average number of Leucaspius eggs laid by plate during the studied period.

Figure 6 : Evolution de la proportion d'œufs d'ables embryonnés sur les plaques en étang, et éclos en aquarium, au cours de la période d'étude.

Figure 6 : Evolution of the rate of Leucaspius eggs embryoned on the plates in pond, and hatched in aquarium, during the studied period. 


\section{Structure de la population}

En fin de période d'étude, des classes de poids de $0,1 \mathrm{~g}$ ont permis de séparer les jeunes de l'année des géniteurs. Parmi les 2.180 ables récoltés en août (fig. 7a) :

- 111 étaient les adultes de l'année précédente (plus de $1,8 \mathrm{~g}$ et plus de $60 \mathrm{~mm}$ ). En effet, leurs écailles montraient un arrêt de croissance hivernal.

- 2.069 étaient des jeunes $0+$ issus de la fraie $(0,1$ à 1,0 g et 18 à $50 \mathrm{~mm})$. Les écailles ne présentaient pas de marque hivernale. Rangés par classes de tailles de $3 \mathrm{~mm}$, trois cohortes d'alevins sont apparues (fig. 7b).

FIGURE 7a :

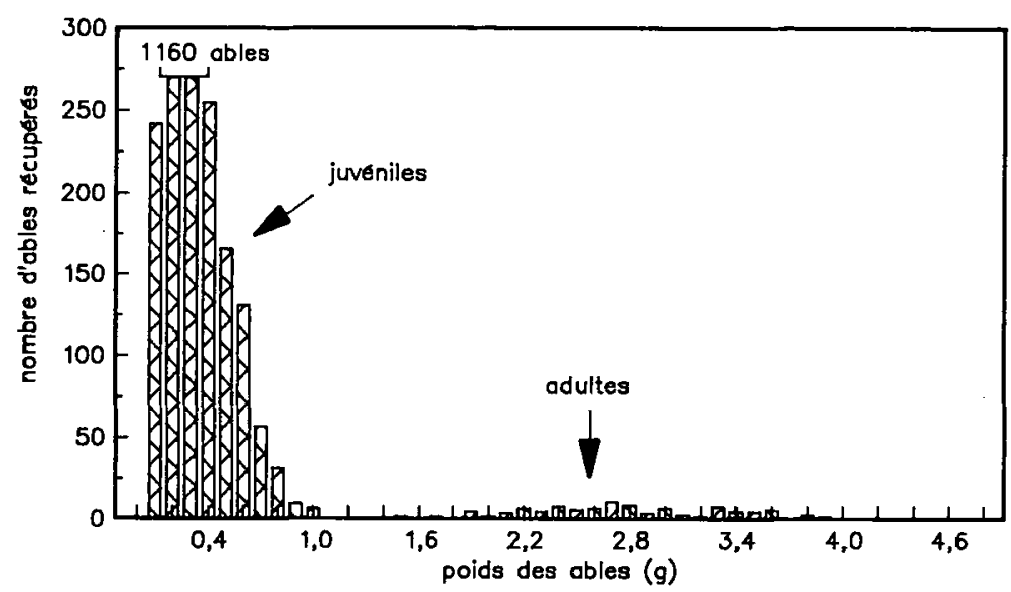

FIGURE 7b :

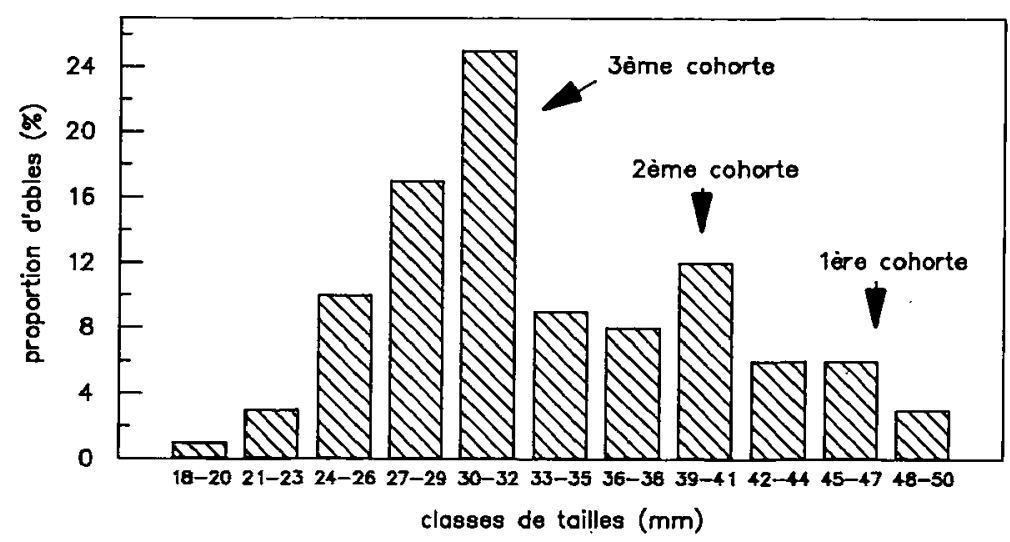

Figure 7a :Structure numérique de la population d'ables en fin d'expérience par classes de poids.

7b : Proportion d'ables 0+ (issus du recrutement de 1990) en fin d'expérience par classes de tailles.

Figure 7a:Numerical structure of the Leucaspius population at the end of the experience, per weight classes.

$7 \mathrm{~b}:$ Leucaspius 0 + proportion (issued from the recrutement of 1990 ) at the end of the experience per length classes. 


\section{DISCUSSION}

Les frayères artificielles construites pour cette étude se sont révélées réellement efficaces à condition d'éliminer systématiquement tout autre support : ainsi aucun œuf n'a été observé dans une autre partie de l'étang.

Pendant la saison de reproduction étudiée, chaque femelle possède toujours les 3 types d'ovocytes, à différents stades d'incorporation du vitellus, qui sont remplacés après leur transformation et leur libération. Le dépôt des œufs par les ables sur les frayères s'étale sur plusieurs mois, ce qui ajouté aux observations histologiques confirme l'hypothèse de pontes multiples pour une même femelle.

Ce phénomène a déjà été étudié pour d'autres Cyprinidés : la carpe Cyprinus carpio possède au même moment des ovocytes à différents stades, leur développement étant d'abord synchrone puis asynchrone (HORVATH, 1986). La tanche Tinca tinca, dont les gonades contiennent plusieurs classes de diamètres d'ovocytes, a des fraies multiples séparées par des périodes de quiescence sexuelle (BRETON et al., 1980). Le goujon Gobio gobio dépose également ses œufs en plusieurs fois, à des intervalles de 15 jours (KESTEMONT, 1990). Mais d'autres poissons n'appartenant pas à cette famille présentent également une ponte fractionnée asynchrone : ainsi le merlu Merluccius merlucciuslibère ses ovocytes en 4 à 5 émissions (SARANO, 1984).

Mi-mai, d'après la structure des gonades, les poissons semblent prêts à la ponte, mais le taux élevé de basophiles diminue sans apparition de vitellus. Un phénomène d'atrésie est constaté, qui peut correspondre à un stress durant la période de pré-fraie et qui retarde l'ovulation. Les causes et la signification de ce processus sont encore mal connues (GURAYA, 1986), mais le $\mathrm{pH}$ anormalement important est soupçonné.

Il est indispensable de tenir compte des paramètres du milieu, la reproduction étant stimulée ou inhibée par des facteurs environnementaux comme la température, la photopériode, la disponibilité de nourriture et la qualité de l'eau (BILLARD et al., 1981) :

Les fortes valeurs de $\mathrm{pH}$ en début de printemps (momentanément plus de 11) peuvent avoir eu un impact sur la survie des ables, alors qu'une eau acide aurait diminué la fertilité des géniteurs et la fécondité des œufs (BILLARD et al.,1981).

Le niveau de température et ses changements sont les facteurs physiques les plus importants dans le contrôle de la reproduction de Cyprinidés vivant en zones tempérées (BILLARD et al., 1981). Le lien entre la date de fraie et un seuil de température s'explique ainsi selon BRETON etal. (1980) : l'accroissement de chaleur stimule la sécrétion d'hormones gonadotropes hypophysaires chez les Cyprinidés et a donc un rôle déterminant dans le déclenchement des différentes phases du cycle de reproduction. De cette façon, le nombre de fraies augmente au cours de la saison (GILLET, 1989).

Le taux d'éclosion en aquarium n'est important (50\%) que lors des premières pontes, sans que les paramètres étudiés soient différents par la suite. Les résultats sont influencés par une mortalité parfois non négligeable, due à la prolifération d'algues et de champignons.

D'autres. facteurs doivent entrer en jeu, qui n'ont pas été analysés, entre autres la pollution venant de la rivière (dont des traces ont été remarquées : particules en suspension). Ils ont pu influencer autant les résultats in situ que dans les aquariums, puisque la même eau était utilisée. Or de nombreux polluants ont des effets néfastes sur la gamétogénèse et la reproduction (ZALEWSKI, 1979).

Aux 3 maxima de pontes correspondent les 3 cohortes de jeunes observées. D'après la vidange finale de l'étang, il reste très peu d'alevins issus des pontes initiales à fort taux d'éclosions et qui constituent la première cohorte ; alors que BREZEANU (1968) avait remarqué une survie remarquable du frai. Mis à part la mortalité naturelle, ils doivent être victimes des insectes prédateurs et des onze brochets présents accidentellement dans l'étang. 


\section{CONCLUSION}

La ponte est bien de type fractionnée asynchrone, puisque chaque ovaire mature contient tous les stades d'ovocytes, que le rapport gonado-somatique est stable, et qu'il y a plusieurs maxima de ponte dans l'année. Cette stratégie permet l'obtention de jeunes même si les conditions sont néfastes durant une partie de la saison de reproduction.

Pour l'année 1990, les œufs ont été pondus à partir du mois de mai, lorsque la température atteignait $20^{\circ} \mathrm{C}$, jusqu'au mois de juillet quand les gonades commençaient à régresser.

L'able semble avoir les caractéristiques d'un excellent poisson-fourrage, pour le brochet par exemple, grâce à l'étalement de son recrutement dans le temps évitant à la population d'être littéralement décimée par un prédateur. En laissant les plantes aquatiques croître afin de ménager des abris, un bon équilibre dans l'étang et un élevage avec un minimum de maind'œuvre sont envisageables.

\section{REMERCIEMENTS}

Nous tenons à remercier Madame Chantal CAUTY du laboratoire de "Physiologie des poissons", pour ses conseils en histologie.

\section{BIBLIOGRAPHIE}

BAGLINIÈRE J.L., LE LOUARN H., 1987. Caractéristiques scalimétriques des, principales espèces de poissons d'eau douce de France. Bull. Fr. Pêche Piscic., 306, 1-39.

BILLARD R., BRY C., GILLET C., 1981. Stress, Environment and Reproduction in Teleost Fish. In Stress and Fish, A.D. Pickering (Ed.), Academic Press, 185-208.

BRETON B., HOROSZEWICZ L., BIENIARZ K., EPLER P., 1980. Temperature and reproduction in tench : effect of a rise in the annual temperature regime on gonadotropine level, gametogenis and spawning. II. The female. Reprod. Nutr. Develop., 20 (4A), 1011-1024.

BREZEANU G.H., 1968. Contributions to the study of the reproduction, prolificity, and development of the species Leucaspius delineatus (Haeckel) (Pisces, Cyprinidae). Trans. Mus. Inst. Nat. gr. Antipa, 8, 509-521.

GABE M., 1968. Techniques histologiques. Masson et Cie, Paris, 1113 p, 232-238.

GILLET C., 1989. Le déroulement de la fraie des principaux poissons lacustres. Hydroécol. Appl., 1/2, 117-193.

GURAYA S.S., 1986. The Cell and Molecular Biology of Fish Oogenesis. Karger (Ed.), Paris, 1-58.

HORVATH L., 1986. Carp oogenesis and the environment. In Aquaculture of Cyprinids, R. Billard et J. Marcel (Ed.), INRA Paris.

KARTAS F., QUIGNARD J.P., 1984. La fécondité des poissons Téléostéens. Coll. Biol. Milieux Marins, 5, 11-51.

KESTEMONT P., 1990. Dynamic aspects of ovogenesis in an asynchronous fish, the gudgeon Gobio gobio L. (Teleostei, Cyprinidae), under controlled temperature and photoperiod conditions. Aquat. Living Resour., 3, 61-74.

PHILLIPPART J.C., VRAUKEN M., 1983. Atlas des poissons de Wallonie. Cahiers d'Ethologie , appliquée 3 (supp. 1-2), 183-186.

SARANO F., 1984. Cycle ovarien du merlu, Merluccius merluccius, poisson à ponte fractionnée. Rév. Trav. Inst. Pêches marit., 48 (1 et 2), 65-76.

WALLACE A., SELMAN., 1981. Cellular and Dynamic Aspects of Oocyte Growth in Teleosts. Amer. Zool., 21, 325-343. 
Bull. Fr. Pêche Piscic. (1991) $320: 109-120-120-$

ZALEWSKI M., 1979. The effect of pollution on the fecundity of roach Rutilus rutilus L., from river of barbel region. 3 rd Europ. Ichtyol. Cong., Warszawa, 18-208.

ZOHAR Y., 1982. L'évolution de la pulsatilité et des cycles nycthéméraux de la sécrétion gonadotrope chez la truite arc-en-ciel femelle, en relation avec le cycle sexuel annue et par rapport à l'activité stéroïdogène de l'ovaire. Thèse doct. état ès Scien. Nat., Univ. Paris VI, 17-22. 